\title{
Neural basis of autobiographical memory retrieval in schizophrenia ${ }^{\dagger}$
}

Christine Cuervo-Lombard, Cédric Lemogne, Fabien Gierski, Céline Béra-Potelle, Eric Tran, Christophe Portefaix, Arthur Kaladjian, Laurent Pierot and Frédéric Limosin

\section{Background}

Autobiographical memory retrieval is impaired in schizophrenia.

\section{Aims}

To determine the neural basis of this impairment.

\section{Method}

Thirteen patients with schizophrenia and 14 healthy controls performed an autobiographical memory retrieval task based on cue words during functional magnetic resonance imaging. Patients were selected on the basis of their ability to perform the task and all participants received training.

\section{Results}

Although patients and controls activated a similar brain network during autobiographical memory retrieval, patients displayed decreased activation in several of these regions, including the anterior cingulate cortex, left lateral prefrontal cortex, right cerebellum and ventral tegmental area $(k \geqslant 10$, $P<0.001$, uncorrected). In addition, activation of the caudate nuclei was negatively correlated with retrieval performance in controls but positively correlated with performance in patients.

\section{Conclusions}

The autobiographical memory retrieval brain network is impaired in schizophrenia. Patients with schizophrenia display decreased activation of the cognitive control network during retrieval, possibly due to aberrant functioning of the dorsal striatum.

\section{Declaration of interest}

C.L. has received speakers fees from AstraZeneca, BristolMyers Squibb, Euthérapie, Lundbeck, Sanofi Aventis and

Pfizer. C.B.-P. has received speakers fees from Bristol-Myers Squibb. E.T. has received speakers fees from Bristol-Myers Squibb, Euthérapie, Janssen-Cilag and Lundbeck. A.K. has received speakers fees from AstraZeneca, Euthérapie, Janssen-Cilag, Lilly and Lundbeck. F.L. has received consulting/speakers fees from Bristol-Myers Squibb, Euthérapie, Janssen-Cilag and Lilly.
Autobiographical memory retrieval, which allows us to re-live subjective experiences from the past, ${ }^{1}$ is impaired in schizophrenia. Compared with healthy individuals, individuals with schizophrenia are less able to retrieve specific events (i.e. events that occurred only once, at a particular time and place, and lasted less than a day $)^{2-4}$ and achieve conscious recollection. ${ }^{5,6}$ People with schizophrenia are also less able to retrieve self-defining events $^{7,8}$ and less prone to draw insight from these memories to inform their sense of identity. ${ }^{9}$ Furthermore, they may not demonstrate the expected oversampling of memories from late adolescence and early adulthood. ${ }^{8,10}$ Taken together with impairments in episodic future thinking, ${ }^{4}$ these deficits may represent a global impairment of the 'mental time travel' ability that allows for the pursuit of long-term goals through coherent decision-making over time. ${ }^{11}$ However, although autobiographical memory retrieval impairment may contribute to the poor social outcomes associated with schizophrenia, ${ }^{12}$ little is known about the neural basis of autobiographical memory retrieval in schizophrenia. In healthy individuals, retrieval entails the activation of an extensive brain network encompassing the cortical midline structures, left lateral prefrontal cortex, medial and lateral temporal lobes, angular gyri and occipital lobes. ${ }^{13,14}$ In the present study, we used functional magnetic resonance imaging (fMRI) to examine the neural basis of autobiographical memory retrieval in schizophrenia. Two meta-analyses of research on episodic memory retrieval in schizophrenia ${ }^{15,16}$ have reported decreased activation of the left lateral prefrontal cortex. Given the role of the left lateral prefrontal cortex in both episodic and autobiographical memory retrieval, ${ }^{13,14}$ we expected to see decreased activation of this region

†See editorial, pp. 423-424, this issue. in people with schizophrenia during the retrieval of specific personal events prompted by cue words.

\section{Method}

\section{Participants}

Seventeen men with a DSM-IV-TR ${ }^{17}$ diagnosis of paranoid schizophrenia were approached to take part in the study. They were either out-patients or in-patients recruited just before discharge and all were deemed to be clinically stable by their psychiatrist (i.e. needing no further adjustment to their treatment). None had any psychiatric comorbid conditions. Thirteen patients were selected on the basis of their ability to complete the verbal tests of the third edition of the Wechsler Adult Intelligence Scale ${ }^{18}$ and the autobiographical memory task (see below). Patients were mildly to moderately ill, according to their mean scores on the Positive and Negative Syndrome Scale (PANSS), ${ }^{19}$ and all were receiving medication (Table 1 ). The mean dose of antipsychotics was equivalent to $375 \mathrm{mg} /$ day (s.d. $=203$ ) of chlorpromazine. ${ }^{20}$ Fourteen healthy men were recruited from the community through advertisements and matched with the patients for age and level of education. None had any current or past psychiatric diagnosis, according to the Mini International Neuropsychiatric Interview 5.0 for DSM-IV Axis I disorders ${ }^{21}$ or any first-degree relative with psychosis and none were on medication. Patients and controls did not differ in age, level of education or verbal abilities (Table 1).

All participants were screened for a history of head trauma, neurological disorder, substance-related disorder, electroconvulsive therapy or claustrophobia. Participants also conformed to 
Table 1 Sociodemographic and clinical characteristics of participants

\begin{tabular}{|c|c|c|c|}
\hline & Patients $(n=13)$ & Controls $(n=14)$ & $P$ \\
\hline Age, years: mean (s.d.) & $30.7(5.3)$ & $30.1(5.0)$ & 0.784 \\
\hline Education level, years: mean (s.d.) & $11.8(2.3)$ & $12.1(1.4)$ & 0.761 \\
\hline Verbal Comprehension Index (WAIS-III), mean (s.d.) & $98.7(15.4)$ & $98.7(12.0)$ & 0.997 \\
\hline Information & $10.1(4.1)$ & $9.0(2.0)$ & 0.400 \\
\hline Similarities & $10.3(2.7)$ & $11.4(3.5)$ & 0.357 \\
\hline Vocabulary & $8.9(3.2)$ & $9.0(1.9)$ & 0.879 \\
\hline Onset of illness, years: mean (s.d.) & $20.2(3.6)$ & & \\
\hline Duration of illness, years: mean (s.d.) & $10.5(3.7)$ & & \\
\hline \multicolumn{4}{|l|}{ Positive and Negative Syndrome Scale, mean (s.d.) } \\
\hline Positive score & $12.9(3.2)$ & & \\
\hline Negative score & $23.0(4.0)$ & & \\
\hline Global psychopathology score & $31.6(5.2)$ & & \\
\hline Total score & $67.5(6.9)$ & & \\
\hline \multicolumn{4}{|l|}{ Psychotropic drugs, $n(\%)$} \\
\hline Risperidone & $8(61.5)$ & & \\
\hline Olanzapine $^{a}$ & $3(23.1)$ & & \\
\hline Haloperidol & $2(15.4)$ & & \\
\hline Cyamemazine $^{a}$ & $1(7.7)$ & & \\
\hline Antidepressants $^{\mathrm{b}}$ & $5(38.5)$ & & \\
\hline Benzodiazepines $^{\mathrm{C}}$ & $3(23.1)$ & & \\
\hline \multicolumn{4}{|l|}{ Marital and housing status, $n(\%)$} \\
\hline Living as a couple & $1(7.7)$ & $8(57.1)$ & \\
\hline Single, living alone & $4(30.8)$ & $5(35.7)$ & \\
\hline Single, living with family & $6(46.2)$ & $1(7.1)$ & \\
\hline Single, living in a therapeutic home setting & $2(15.4)$ & & \\
\hline \multicolumn{4}{|l|}{ Employment status, $n$ (\%) } \\
\hline Student & $1(7.7)$ & & \\
\hline Working in a normal setting & $2(15.4)$ & $14(100)$ & \\
\hline Working in a therapeutic setting & $1(7.7)$ & & \\
\hline Not working & $9(69.2)$ & & \\
\hline \multicolumn{4}{|c|}{$\begin{array}{l}\text { WAIS-III: Wechsler Adult Intelligence Scale (3rd edn). } \\
\text { a. One patient was taking both olanzapine and cyamemazine, the latter being used as an anxiolytic. } \\
\text { b. Three patients were taking paroxetine ( } 20 \mathrm{mg} / \text { /day), one fluoxetine }(20 \mathrm{mg} / \text { day) and one venlafaxine }(112.5 \mathrm{mg} / \text { day). } \\
\text { c. Two patients were taking clorazepate }(40 \text { and } 60 \mathrm{mg} / \text { day) and one prazepam ( } 40 \mathrm{mg} / \mathrm{day}) \text {. }\end{array}$} \\
\hline
\end{tabular}

standard health and safety regulations regarding the use of magnetic resonance imaging (MRI). All participants were righthanded, according to the Edinburgh Handedness Inventory, ${ }^{22}$ and were native French speakers. This study was approved by the local ethics committee and all participants gave their written informed consent after receiving a complete description of the study.

\section{Experimental design}

During the fMRI session, 25 cue words and 25 control words were presented in a pseudorandomised order such that words of each type (cue $v$. control) followed each other equally often. The cue words (for example chat (cat), école (school), voiture (car)) were French nouns selected from previous studies on the basis of their frequency in the spoken language and their ability to elicit vivid mental images. ${ }^{23,24}$ They were displayed for $12 \mathrm{~s}$ and followed by a fixation cross for $4 \mathrm{~s}$. This fairly long duration was chosen to allow patients and controls sufficient time to recall a specific event (i.e. a personal event that had occurred only once, at a particular time and place, and lasted less than a day ${ }^{25}$ ). The control words were either 'index' or 'middle'. They were displayed for $6 \mathrm{~s}$ and followed by a fixation cross for $4 \mathrm{~s}$. This shorter time was chosen to prevent mind-wandering. All the stimuli were shown on an MRI-compatible monitor (white text, black background) viewed by participants via a mirror mounted on the head coil. We used E-Prime software for Windows (Psychology Software Tools, Sharpsburg, Pennsylvania, USA, www.pstnet.com/) to present the stimuli and collect reaction times. Responses were made on an MRI-compatible response pad. For autobiographical memory retrieval, the participants had to press the button under their right middle finger as soon as a memory was fully formed in their mind. For the control words, they had to press the button under the appropriate finger (i.e. index $v$. middle).

Immediately after leaving the scanner, participants had to describe each of their memories with as much detail as possible regarding the content of the event (what), where it took place (where) and any temporal information about it (when). They then had to indicate their age at the time of the event (i.e. encoding age) and the emotional valence of the memory on a $10 \mathrm{~cm}$ visual scale (ranging from 'most unpleasant' to 'most pleasant'). In addition, they had to indicate their subjective state of consciousness during autobiographical memory retrieval for each aspect of the memory (i.e. what, where and when). ${ }^{26}$ The participants gave either a 'Remember' response if they recollected phenomenological aspects such as perceptions, thoughts or feelings that were experienced at the time of the event, a 'Know' response if retrieval was achieved without any such recollection or a 'Guess' response if retrieval was doubtful. ${ }^{6,26,27}$ A recollection score ranging from zero to three was calculated for each memory, based on the total number of 'Remember' responses for the three different aspects (i.e. what, where and when). No memory and thus no conscious recollection was rated zero. Finally, the participants were fully debriefed.

To ensure that the participants were able to perform the autobiographical memory retrieval task properly, including the remember/know paradigm, we ran a training session in a quiet room 1 week before the fMRI session and after a full explanation of the task. This training session featured a list of seven cue words that were not used in the subsequent fMRI session. The participants had to recall a specific event for each cue word and 
immediately describe and assess each memory (see above). To familiarise the participants with the MRI environment (MRI table, screen, response pad) and the experiment's timing constraints, a second training session took place at the beginning of the fMRI session, when participants were already on the MRI table. Two other cue words and three control words were used.

\section{Memory specificity assessment}

The specificity of each memory (transcribed verbatim) was assessed by two independent raters (C.C.L. and Sarah Barrière) on a four-point scale: ${ }^{25} 4$ for a highly specific event located in time and place, described with numerous details; 3 for a specific event located in time and place, but not detailed; 2 for a repeated event; 1 for a vague event; 0 for no memory or only general information about a theme. One of the raters (Sarah Barrière) was masked to the diagnosis. Cohen's kappa coefficient indicated substantial interrater agreement $(\kappa=0.94, P<0.001)$. Every conflicting rating was re-examined until a consensus was reached. For each patient, a mean specificity score was computed. Together with the recollection score, this specificity score was used to index individual autobiographical memory retrieval performances.

\section{MRI data acquisition}

Imaging was conducted with a $3 \mathrm{~T}$ whole-body MRI scanner with an eight-channel head coil. Head motions were minimised with a forehead strap and comfortable padding around the participant's head. For each participant, a $T_{1}$-weighted anatomical image, oriented parallel to the anterior commissure-posterior commissure axis was first acquired with a fast-field echo (FFE) sequence $\left(T_{1}\right.$-FFE; repetition time $(\mathrm{TR})=253 \mathrm{~ms}$; echo time $(\mathrm{TE})=2.30 \mathrm{~ms}$; flip angle: $80^{\circ}$; 32 -axial slices; slice thickness: $4.50 \mathrm{~mm}$; no gap; field of view $(F O V)=240 \times 240 \mathrm{~mm}^{2}$; matrix: $268 \times 214$; acquisition voxel size: $0.43 \times 0.43 \times 4.5 \mathrm{~mm}^{3}$ ). Functional data were acquired in ascending slice order with a two-dimensional (2D) $T_{2}{ }^{*}$-weighted echo-planar imaging (EPI) sequence sensitive to blood oxygen level-dependent (BOLD) contrast in the same axial plane as the $T_{1}$-weighted structural images (2D $T_{2}{ }^{*}$-FFE-EPI; $\mathrm{TR}=2000 \mathrm{~ms} ; \mathrm{TE}=33 \mathrm{~ms}$; flip angle: $90^{\circ} ; 32$-axial slices; slice thickness: $4.5 \mathrm{~mm}$; no gap; matrix: $80 \times 80 ; \quad F O V=240 \times$ $240 \mathrm{~mm}^{2}$; acquisition voxel size: $3 \times 3 \times 4.5 \mathrm{~mm}^{3}$ ). The functional volumes were collected during a single functional session (330 volumes). Finally, a high-resolution $T_{1}$-weighted anatomical image was acquired with a three-dimensional (3D) turbo-field echo (TFE) sequence ( $3 \mathrm{D} T_{1}$-TFE, $\mathrm{TR}=8.2 \mathrm{~ms}$; $\mathrm{TE}=3.73 \mathrm{~ms}$; flip angle: $8^{\circ}$; 160 axial slices; slice thickness: $1 \mathrm{~mm}$; FOV $=240 \times 240 \mathrm{~mm}^{2}$; matrix: $240 \times 240$; acquisition voxel size: $1 \times 1 \times 1 \mathrm{~mm}^{3}$ ).

\section{MRI image pre-processing}

The fMRI data were analysed with Statistical Parametric Mapping (SPM8; Wellcome Department of Cognitive Neurology, Institute of Neurology, London, UK, www.fil.ion.ucl.ac.uk/spm/) on Windows. MRIcro software for Windows (www.mccauslandcenter.sc.edu/ mricro/mricro) was used for image conversion. Five initial brain volumes were discarded from the analyses to eliminate the non-equilibrium effects of magnetisation. Functional EPI-BOLD images were spatially realigned to the first volume. There were no patient or control images with excessive head movements $\left(>2 \mathrm{~mm}\right.$ or $>2^{\circ}$ ). Interpolation was used to minimise timing errors between slices. These images were then anatomically normalised to the Montreal Neurological Institute (MNI) template and smoothed with an isotropic Gaussian kernel of $8 \mathrm{~mm}$ full-width at half maximum. A high-pass filter was implemented, using a cut-off period of $128 \mathrm{~s}$, to remove lowfrequency drift from the time series.

\section{fMRI statistical analysis}

Individual analyses were performed with a fixed-effect model, whereas group analyses were performed with a random-effect model. For each participant, we computed an individual statistical parametric map based on the general linear model and an eventrelated approach. ${ }^{28}$ Since our main hypothesis was predicated on previous fMRI studies of episodic retrieval, in which search processes may prevail over other processes such as mental imagery, our primary aim was to investigate the search component of autobiographical memory retrieval. We thus computed a convolution of each stimulus onset with the canonical haemodynamic response function to create regressors of interest. Motion parameters (three translations and three rotations) determined from the initial realignment procedure were included as regressors of non-interest. Statistical parametric maps of $t$-statistics were calculated to identify voxels with event-related signal changes. The resulting $t$-statistics were transformed to $z$-score maps of normal unit distribution. As regards the main effect of the task (i.e. autobiographical memory retrieval $v$. control), we used a family-wise error (FWE) corrected threshold of $P<0.05$. To look for between-group differences in the brain regions actually involved in autobiographical memory retrieval, we first defined an inclusive mask including all regions activated during retrieval in either patients or controls, separately, with a statistical threshold of $P<0.05$, FWE-corrected. ${ }^{29}$ We then looked for between-group differences within these regions, combining an extent threshold of 10 voxels, with a statistical threshold of $P<0.001$, uncorrected.

\section{Results}

\section{Behavioural results}

The groups did not differ on the quantity of memories, their specificity or the recollection scores. Nor did they differ on remoteness, encoding age or emotional valence (Table 2). Discrepancies between behavioural responses during fMRI and the post-scanning assessment revealed that 8 participants (5 patients, 3 controls) forgot at least once to press the button after recalling a personal event, whereas 14 participants ( 6 patients, 8 controls) pressed it at least once even though they had not recalled a personal event. Behavioural responses and reaction times were thus discarded from subsequent analyses.

\section{fMRI results}

\section{A priori analyses}

Regardless of group, autobiographical memory retrieval activated an extensive brain network including the cortical midline structures, left lateral prefrontal cortex, left angular gyrus, medial and lateral temporal lobes, occipital lobes and cerebellum (Table 3 and Fig. 1). To identify the regions activated in both patients and controls, we performed a conjunction analysis with a conjunction null hypothesis (i.e. an 'AND' conjunction ${ }^{29}$ ), which yielded a smaller number of activation clusters (Table 4 ).

Since we were primarily interested in examining between-group differences in the regions actually involved in autobiographical memory retrieval, we then performed a conjunction analysis with a global null hypothesis (i.e. an 'OR' conjunction) to identify regions that were activated during retrieval in either patients or controls. ${ }^{29}$ This map was used as an inclusive mask to look for between-group differences. Although none of these regions was 
Table 2 Autobiographical memory task: behavioural results

\begin{tabular}{|c|c|c|c|c|}
\hline & \multirow{2}{*}{$\begin{array}{l}\text { Patients, mean (s.d.) } \\
\qquad(n=13)\end{array}$} & \multirow{2}{*}{$\begin{array}{l}\text { Controls, mean (s.d.) } \\
\qquad(n=14)\end{array}$} & \multicolumn{2}{|c|}{ Student's $t$-test } \\
\hline & & & $t_{25}$ & $P$ \\
\hline Number of memories, maximum 25 & $16.85(6.15)$ & $17(4.90)$ & -0.07 & 0.94 \\
\hline Specificity score, maximum 4 & $1.81(0.66)$ & $2.17(0.68)$ & -1.44 & 0.16 \\
\hline Recollection score, maximum 3 & $1.46(0.59)$ & $1.47(0.49)$ & -0.005 & 0.98 \\
\hline Emotional valence, pleasantness \% & $64.57(11.78)$ & $60.30(12.78)$ & 0.90 & 0.38 \\
\hline Remoteness, years & $10.82(5.24)$ & $11.34(7.20)$ & 0.50 & 0.62 \\
\hline Encoding age, year & $19.87(4.84)$ & $18.81(6.14)$ & -0.21 & 0.83 \\
\hline
\end{tabular}

\begin{tabular}{|c|c|c|c|c|c|}
\hline \multirow[b]{2}{*}{ Brain regions } & \multirow[b]{2}{*}{$\mathrm{mm}^{3}$} & \multirow[b]{2}{*}{$z$-score at peak voxel } & \multicolumn{3}{|c|}{ MNI coordinates at peak voxel } \\
\hline & & & $x$ & $y$ & $z$ \\
\hline $\begin{array}{l}\text { Precuneus (L/R), lingual and parahippocampal gyri } \\
(\mathrm{L} / \mathrm{R}) \text {, midbrain }(\mathrm{L} / \mathrm{R})\end{array}$ & 49752 & Inf & -4 & -54 & 20 \\
\hline Frontal inferior gyrus $(\mathrm{L})$ & 55584 & 7.39 & -50 & 22 & 12 \\
\hline Cerebellum (R) & 2408 & 6.75 & 12 & -82 & -24 \\
\hline Frontal inferior gyrus (R) & 2592 & 6.48 & 34 & 28 & -6 \\
\hline Cerebellum (R) & 2672 & 6.41 & 36 & -66 & -32 \\
\hline Temporal inferior and middle gyri (L) & 760 & 6.06 & -54 & -44 & -16 \\
\hline Cerebellum (L) & 1664 & 6.03 & 4 & -58 & -38 \\
\hline Angular gyrus (L) & 3520 & 5.95 & -42 & -74 & 34 \\
\hline Frontal medial orbital gyrus (L) & 512 & 5.34 & -8 & 56 & -6 \\
\hline Cerebellum (L) & 376 & 5.23 & -38 & -58 & -36 \\
\hline Occipital inferior and middle gyri (L), calcarine sulcus (L) & 560 & 5.20 & -28 & -98 & 2 \\
\hline Calcarine sulcus (R) & 168 & 5.00 & 18 & -100 & 4 \\
\hline
\end{tabular}

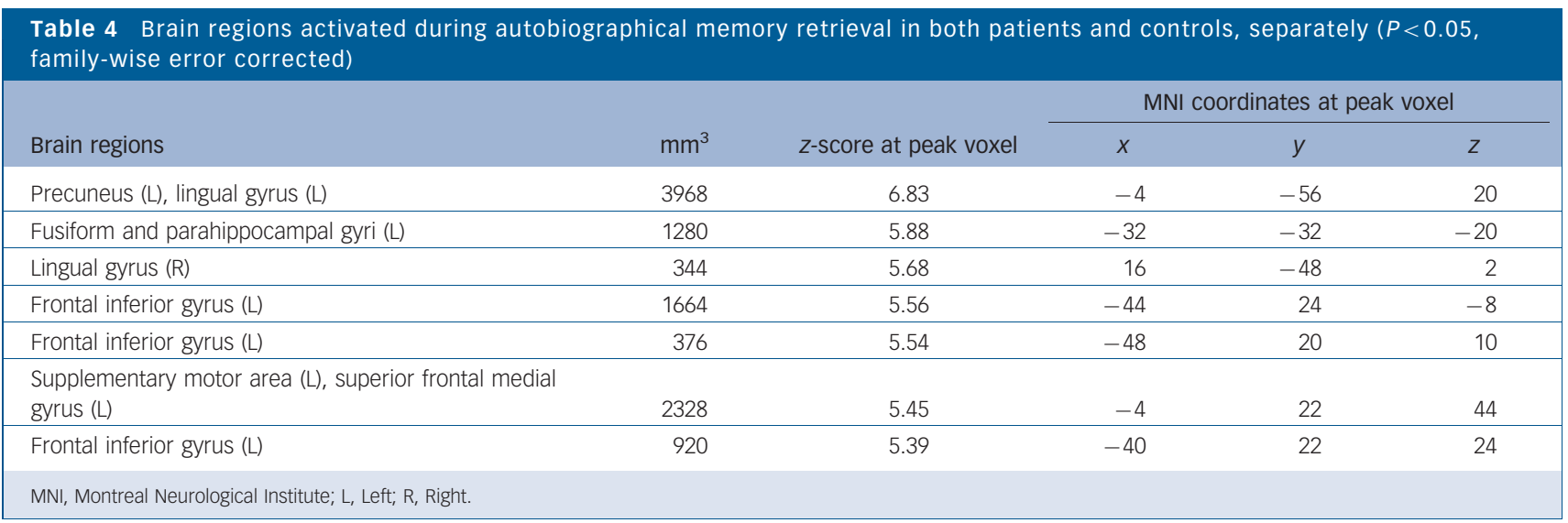

more activated in patients than controls $(P>0.001$, uncorrected $)$, several of them were less activated in patients than controls, including some cortical midline structures (i.e. the anterior cingulate cortex and the precuneus), the left lateral prefrontal cortex, left medial temporal lobe, occipital lobe, cerebellum and a midbrain region extending to the lateral ventral tegmental area (Table 5 and Fig. 2). Adjusting for autobiographical memory retrieval performances yielded similar results except for the left medial temporal lobe, whose activation was no longer significant when adjusting for either specificity or recollection, and the precuneus, whose activation was no longer significant when adjusting for specificity (see online Tables DS1 and DS2).

We then looked for between-group differences at a wholebrain level, using a more stringent statistical threshold to avoid type I errors due to multiple comparisons. This analysis revealed four clusters of activation corresponding to the left lateral ventral tegmental area $\left(16 \mathrm{~mm}^{3}\right.$, MNI coordinates: $-12,-20,-16$; $z_{(1,25)}=4.93, P<0.05$, FWE-corrected), the right cerebellum $\left(48 \mathrm{~mm}^{3}\right.$, MNI coordinates: $20,-38,-28 ; z_{(1,25)}=4.86$, $P<0.05$, FWE-corrected) and the caudate nuclei $\left(48 \mathrm{~mm}^{3}\right.$, MNI coordinates: $-10,16,6, z_{(1,25)}=4.85$; and $64 \mathrm{~mm}^{3}$, MNI coordinates: $10,18,4, z_{(1,25)}=4.80, P<0.05$, FWE-corrected) (Fig. 3(a)).

\section{Post hoc analyses}

Although the lateral ventral tegmental area and the cerebellum clusters were among the between-group differences that had previously been found within the global null conjunction mask, the caudate nuclei clusters were not. To examine how these two clusters were functionally related to autobiographical memory 

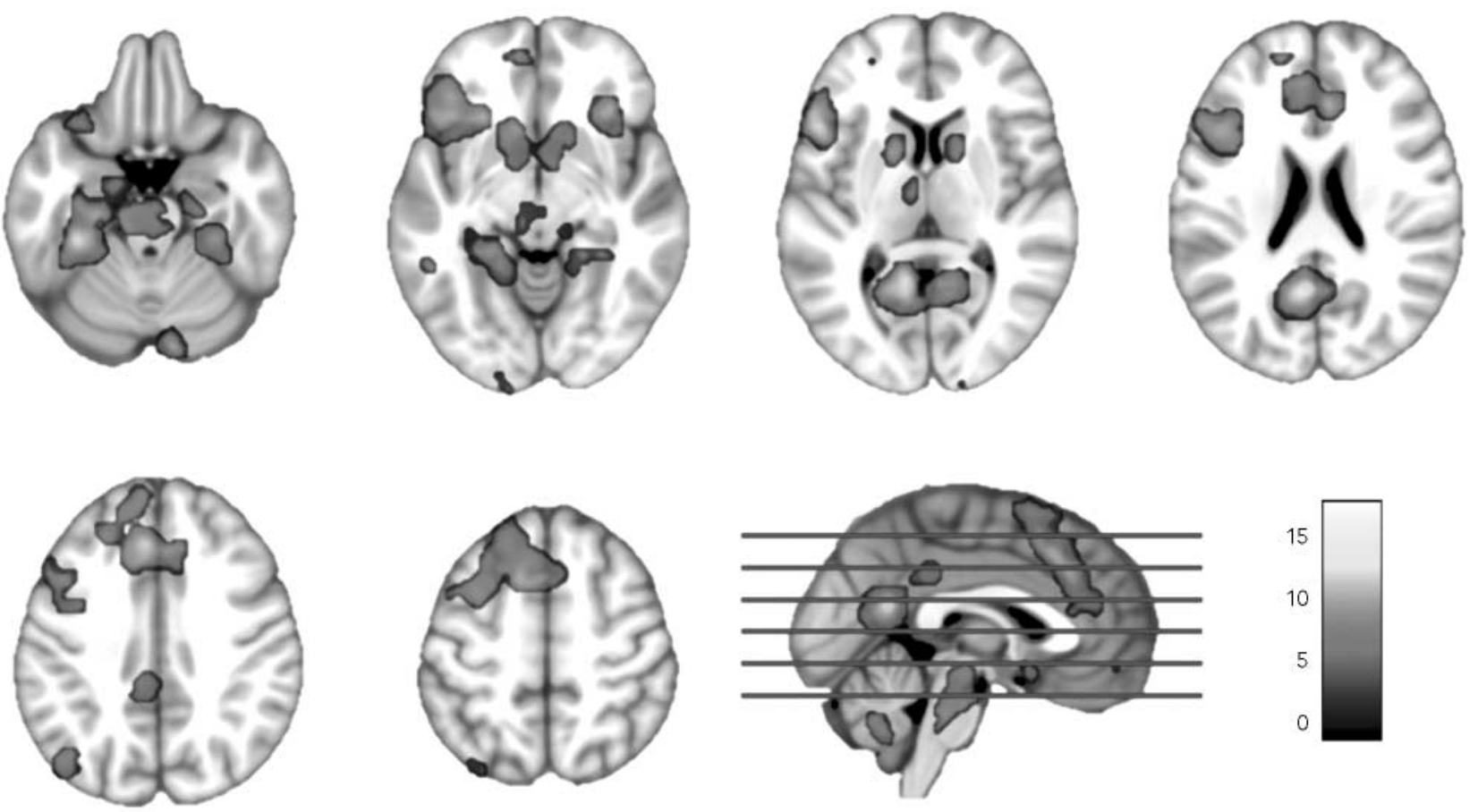

Fig. 1 Brain regions activated during autobiographical memory retrieval regardless of group $(P<0.05$, family-wise error corrected) displayed on a $1 \mathrm{~mm}$ isotropic version of the Montreal Neurological Institute (MNI) 152 standard brain.

See online Fig. DS1 for a colour version of this figure.

retrieval, we looked for correlations between their mean beta values and retrieval performances (i.e. specificity and recollection). The activation of the left caudate nucleus tended to be positively correlated with retrieval performances in patients (specificity: $r=0.705, P=0.007$; recollection: $r=0.589, P=0.034$ ), but negatively with performances in controls (specificity: $r=-0.508, P=0.063$; recollection: $r=-0.367, P=0.196)$, thus yielding significant group $\times$ specificity $(z=3.289, P<0.001)$ and group $\times$ recollection $(z=2.273, P=0.023)$ interactions (Fig. 3(b)). A similar pattern was observed in the right caudate nucleus, although neither the group $\times$ specificity $(z=1.694, P=0.090)$ nor the group $\times$ recollection $(z=1.242, P=0.214)$ interaction reached significance. There was no significant correlation between retrieval performances and the activation of the other regions characterised by between-group differences during retrieval.

Finally, to rule out the potential role of antipsychotic drugs and look for clinical correlations, we performed regression analyses among the patients only, using the between-group differences as an inclusive mask. There was no significant correlation with either the PANSS subscales or the chlorpromazine equivalent dose at a threshold of $P<0.001$, uncorrected. Using a very lenient statistical threshold to avoid type II errors, we found a negative correlation between the chlorpromazine equivalent dose and the activation of the left medial temporal lobe $\left(72 \mathrm{~mm}^{3}\right.$, MNI coordinates: -22 , $-20,-22 ; z_{(11)}=2.13, P<0.05$, uncorrected).

\section{Discussion}

\section{Main results}

This study investigated whether the neural basis of autobiographical memory retrieval differs between patients with schizophrenia and healthy controls. As expected, because patients were selected according to their ability to perform the autobiographical

Table 5 Brain regions displaying greater activation in controls than patients ( $k \geqslant 10, P<0.001$, uncorrected) among those regions activated during autobiographical memory retrieval in either controls or patients $(P<0.05$, family-wise error corrected)

\begin{tabular}{|c|c|c|c|c|c|}
\hline \multirow[b]{2}{*}{ Brain regions } & \multirow[b]{2}{*}{$\mathrm{mm}^{3}$} & \multirow[b]{2}{*}{$z$-score at peak voxel } & \multicolumn{3}{|c|}{ MNI coordinates at peak voxel } \\
\hline & & & $x$ & $y$ & $z$ \\
\hline Frontal inferior gyrus (L) & 720 & 4.48 & -54 & 22 & 6 \\
\hline Ventral tegmental area (L) & 104 & 4.40 & -10 & -20 & -16 \\
\hline Anterior cingulate cortex (L) & 408 & 4.05 & -2 & 42 & 22 \\
\hline Frontal inferior gyrus (R), insula (R) & 232 & 3.95 & 34 & 28 & -8 \\
\hline Cerebellum (R) & 232 & 3.89 & 40 & -66 & -32 \\
\hline Cerebellum (R) & 184 & 3.65 & 16 & -84 & -28 \\
\hline Anterior cingulate cortex (R) & 232 & 3.62 & 4 & 30 & 30 \\
\hline Precuneus (L) & 136 & 3.60 & 0 & -54 & 22 \\
\hline Parahippocampal gyrus (L) & 88 & 3.47 & -22 & -20 & -22 \\
\hline Calcarine sulcus (L), lingual gyrus (L) & 256 & 3.41 & -8 & -52 & 6 \\
\hline
\end{tabular}




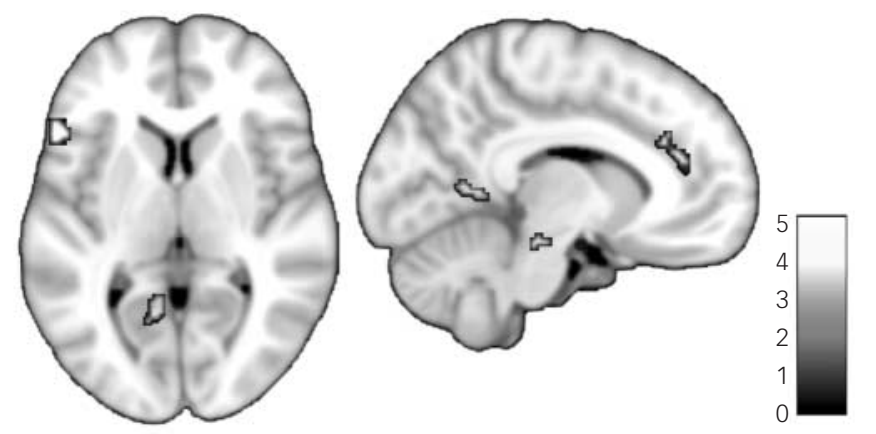

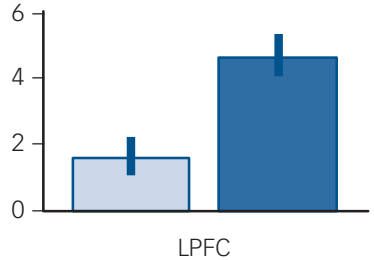

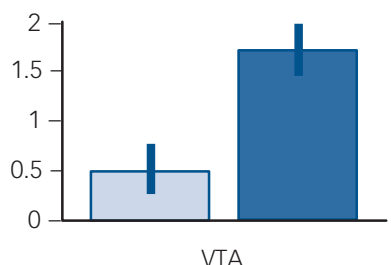

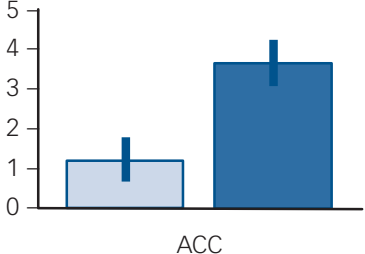

Patients

Controls

Fig. 2 (a) Brain regions displaying greater activation in controls than in patients $(P<0.001$, uncorrected; $k=10)$ among those activated

during autobiographical memory retrieval in either controls or patients $(P<0.05$, family-wise error corrected) displayed on a $1 \mathrm{~mm}$ isotropic version of the Montreal Neurological Institute (MNI) 152 standard brain. (b) Mean contrast estimates and $95 \%$ confidence interval during autobiographical memory retrieval ( $v$. control task) in the lateral prefrontal cortex (LPFC, MNI coordinates: $-54,22,6)$, ACC, anterior cingulate cortex (ACC, MNI coordinates: $-2,42,22$ ) and ventral tegmental area (VTA, MNI coordinates: $-10,-20,-16$ ).

See online Fig. DS2 for a colour version of Fig. 2(a).

memory retrieval task, the retrieval performances measured after the scan were similar in both groups. However, we were not able to measure retrieval performances during the scan. Consistent with previous studies with healthy individuals, ${ }^{13,14}$ patients and controls activated a similar brain network during retrieval, including the cortical midline structures, left lateral prefrontal cortex, left angular gyrus, medial temporal lobes, occipital lobe and cerebellum. Compared with controls, patients with schizophrenia displayed reduced activation in several of these regions, including two cortical midline structures (i.e. the anterior cingulate cortex and precuneus), the left lateral prefrontal cortex, left medial temporal lobe, occipital lobe, right cerebellum and left lateral ventral tegmental area. Apart from the decreased activation of the left lateral prefrontal cortex, which was expected a priori, the other findings should be interpreted with caution because of the uncorrected statistical threshold. In addition, a whole-brain analysis with a more stringent statistical threshold revealed between-group differences outside these regions, especially in the caudate nuclei.

Given the functional neuroanatomy of autobiographical memory retrieval in healthy individuals, ${ }^{13,14}$ the present results may reflect a global impairment of retrieval in patients with schizophrenia, encompassing strategic search (left lateral prefrontal cortex, anterior cingulate cortex, right cerebellum), self-referential processing (cortical midline structures), conscious recollection (medial temporal lobe, precuneus) and visual imagery (precuneus, occipital lobe). Apart from the left medial temporal lobe and the precuneus, between-group differences remained significant when adjusting for individual retrieval performances.

\section{Decreased activation of the cognitive control network}

One may argue that focusing on discrete brain regions rather than distributed networks is unlikely to capture the core processes that underlie our findings. First, the neural signature of autobiographical memory retrieval is not unique. ${ }^{30}$ Second, schizophrenia is thought to be characterised by a lack of coordinated activation of segregated neural networks rather than by the aberrant activation of any given region. ${ }^{31}$ Among healthy individuals, the neural basis of episodic memory retrieval are thought to involve three segregated yet interacting networks. ${ }^{32}$ First, the so-called 'default-mode network', including the precuneus and the medial temporal lobe, may underlie self-referential processing and conscious recollection. Second, a dorsal frontoparietal network, including the anterior cingulate cortex and the lateral prefrontal cortex, may underlie increased top-down cognitive control, such as that triggered by difficulty achieving retrieval goals. Third, a ventral frontoparietal network may underlie bottom-up salience processing, as a complementary system for guiding memory searches.

Our results suggest that autobiographical memory retrieval in schizophrenia can be mainly characterised by decreased activation of a cognitive control network involving the left lateral prefrontal cortex, dorsal anterior cingulate cortex and right cerebellum, which is functionally and anatomically connected to the left lateral prefrontal cortex. ${ }^{33,34}$ Since our autobiographical memory task was based on generative retrieval (i.e. an effortful constructive process) rather than direct retrieval (i.e. an automatic process triggered by a specific cue provided during a pre-scan interview ${ }^{1}$ ), we believe that our study specifically addressed the controlled aspects of autobiographical memory retrieval. Our results may thus highlight a lack of top-down cognitive control during retrieval, making patients with schizophrenia less able to perform goal-directed memory searches.

\section{Aberrant activation of the dorsal striatum}

Among healthy individuals, the dorsal striatum, including the caudate nuclei, plays a critical role in strategy shifting, through prediction error signalling (i.e. signalling a discrepancy between expected and actual outcome). In the context of memory search, prediction errors during free recall may indicate when to shift from automatic (bottom-up) to controlled (top-down) retrieval strategies. ${ }^{35}$ In the present study, the strongest between-group differences were observed in the caudate nuclei, with greater activation in controls than in patients during autobiographical memory retrieval. Among healthy individuals, the activation of the caudate nucleus is greater during the retrieval of recent $(v$. remote) and positive ( $v$. negative) memories. ${ }^{36,37}$ However, in the present study, encoding age, remoteness and valence did not differ between groups. Moreover, the activation of the left caudate nucleus during autobiographical memory retrieval was inversely correlated with autobiographical memory performances in patients and controls. Increased activation in controls was 


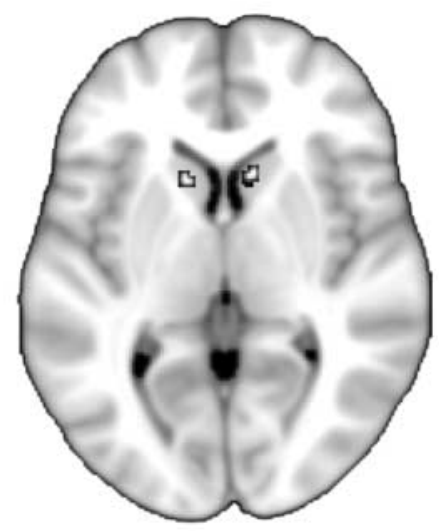

(b)
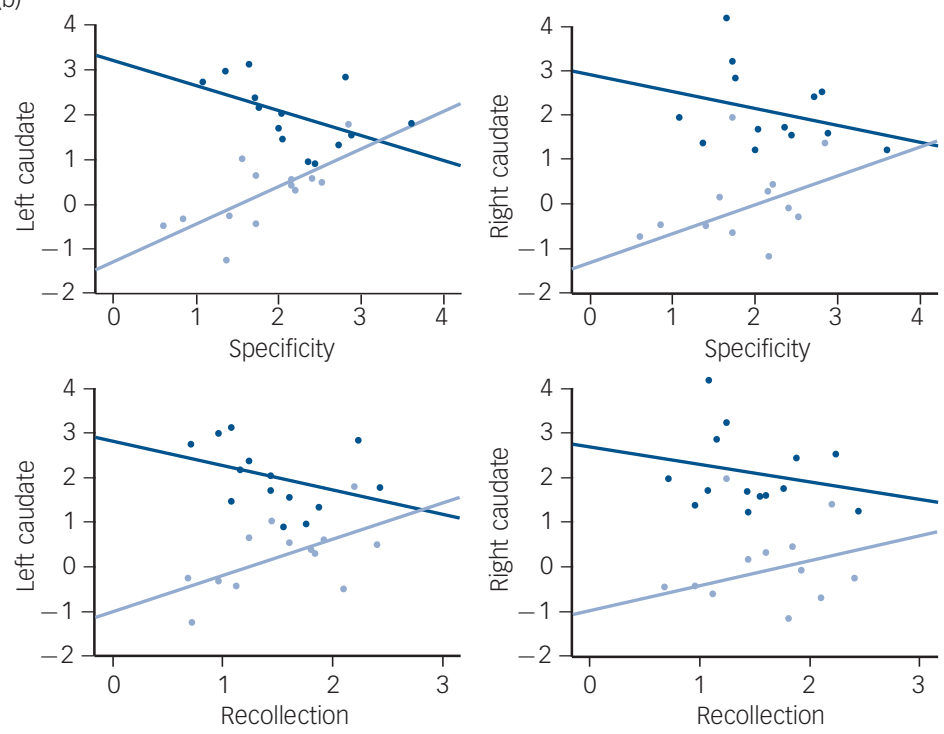

Fig. 3 (a) Greater activation of the caudate nuclei in controls than in patients during autobiographical memory retrieval $(P<0.05$, family-wise error corrected) displayed on a $1 \mathrm{~mm}$ isotropic version of the Montreal Neurological Institute (MNI) 152 standard brain. (b) Correlations between the mean contrast estimates of the two caudate nucleus clusters during autobiographical memory retrieval (v. control) and the specificity and recollection scores among patients (light blue) and controls (dark blue).

See online Fig. DS3 for a colour version of Fig. 3(a).

associated with lower retrieval performances, suggesting that a relative failure of retrieval may have triggered increased cognitive control. In contrast, increased activation in patients was associated with higher autobiographical memory retrieval performances, suggesting that it may represent a partially successful compensatory mechanism. This aberrant dorsal striatum activation may account for the decreased activation of the cognitive network during retrieval in schizophrenia. This hypothesis is consistent with the similar pattern of activation observed in the lateral ventral tegmental area, whose erratic dopaminergic outputs to the striatum may mediate aberrant prediction error in schizophrenia. ${ }^{31,38}$

\section{Limitations}

Several limitations should be acknowledged. First, our sample was fairly small and included only male patients who were selected according to their verbal abilities, thus limiting the generalisability of our results. In addition, all the patients were taking antipsychotic drugs, which may have influenced our results to some extent. However, although we found a weak negative correlation between the chlorpromazine equivalent dose and the activation of the left medial temporal lobe, we found no correlation within the cognitive control network. Second, our results may partially reflect processes shared with other cognitive functions that are impaired in schizophrenia, such as episodic memory retrieval ${ }^{15,16}$ or executive functions. ${ }^{39}$ Third, we were not able to measure autobiographical memory performances during the scan or verify the veracity of the reported memories. Fourth, as we did not include psychiatric patients as controls, we cannot be sure that our findings are specific to schizophrenia. For instance, there is evidence that autobiographical memory retrieval is also impaired in major depression. ${ }^{27}$ Fifth, our experimental design may have magnified the role of top-down attention, as it focused on generative retrieval. ${ }^{1}$ Further studies are therefore needed to examine the role of bottom-up processes, as well as their interplay with top-down processes during autobiographical memory retrieval in patients with schizophrenia. Finally, our study lacked a measure of global functioning that could have been used to examine the functional correlates and clinical significance of our results.

\section{Implications}

Given the role of autobiographical memory in the pursuit of long-term goals and social cognition, ${ }^{1}$ this autobiographical memory retrieval impairment may contribute to poor social outcomes associated with schizophrenia. ${ }^{12}$ Future studies will need to confirm these preliminary results among unmedicated and female patients and translate these results into therapeutic interventions such as cognitive remediation.

Christine Cuervo-Lombard, MSC Psy, PhD, Department of Psychiatry, Centre Hospitalier Universitaire de Reims, Reims and LCPI Laboratory, Toulouse 2 Le Mirail University, Department of Psychology, Toulouse; Cédric Lemogne, MD, PhD, Université Paris Descartes, Sorbonne Paris Cité, Faculté de Médecine, INSERM UMR 894, Centre Psychiatrie et Neurosciences and Assistance Publique-Hôpitaux de Paris, Department of Psychiatry, Hôpitaux Universitaires Paris Ouest, Issy-LesMoulineaux, Paris; Fabien Gierski, MSc Psy, PhD, Department of Psychiatry, Centre Hospitalier Universitaire de Reims, Department of Radiology, Centre Hospitalier Universitaire de Reims and C2S Laboratory (EA6291), Reims Champagne-Ardenne University, Reims; Céline Béra-Potelle, MD, Eric Tran, MD, Department of Psychiatry, Centre Hospitalier Universitaire de Reims, Reims; Christophe Portefaix, PhD, Department of Radiology, Centre Hospitalier Universitaire de Reims, Reims; Arthur Kaladjian, MD, PhD, Department of Psychiatry, Centre Hospitalier

Universitaire de Reims, Reims; Laurent Pierot MD, PhD, Department of Radiology, Centre Hospitalier Universitaire de Reims, Reims; Frédéric Limosin, MD, PhD, Université Paris Descartes, Sorbonne Paris Cité, Faculté de Médecine, INSERM UMR 894, Centre Psychiatrie et Neurosciences and Assistance Publique-Hôpitaux de Paris, Department of Psychiatry, Hôpitaux Universitaires Paris Ouest, Issy-Les-Moulineaux, Paris, France

Correspondence: Cédric Lemogne, Service de Psychologie Clinique et de Psychiatrie de Liaison, Hôpital Européen Georges Pompidou, 20 rue Leblanc 75015 Paris, France. Email: cedric.lemogne@egp.aphp.fr

First received 20 Jul 2011, final revision 25 Nov 2011, accepted 13 Feb 2012

\section{Funding}

This work was funded by a 'Projet Hospitalier de Recherche Clinique' grant (AOL2007) from the Centre Hospitalier Universitaire de Reims. 


\section{Acknowledgements}

We thank Sarah Barrière for the masked assessment of specificity, Marion Lesgourgues for her help in assessing the participants and Jevita Potheegadoo for the English manuscript editing.

\section{References}

1 Conway MA. Memory and the self. J Mem Lang 2005; 53: 594-628.

2 Feinstein A, Goldberg TE, Nowlin B, Weinberger DR. Types and characteristics of remote memory impairment in schizophrenia. Schizophr Res 1998; 30 : $155-63$.

3 Riutort M, Cuervo C, Danion JM, Peretti CS, Salamé P. Reduced levels of specific autobiographical memories in schizophrenia. Psychiatry Res 2003; 117: $35-45$.

4 D'Argembeau A, Raffard S, Van der Linden M. Remembering the past and imagining the future in schizophrenia. J Abnorm Psychol 2008; 117: 247-51.

5 Danion JM, Cuervo C, Piolino P, Huron C, Riutort M, Peretti CS, et al. Conscious recollection in autobiographical memory: an investigation in schizophrenia. Conscious $\operatorname{Cog} 2005$; 14: 535-47.

6 Neumann A, Blairy S, Lecompte D, Philippot P. Specificity deficit in the recollection of emotional memories in schizophrenia. Conscious Cogn 2007 16: $469-84$.

7 Raffard S, D'Argembeau A, Lardi C, Bayard S, Boulenger J, Van Der Linden M. Exploring self-defining memories in schizophrenia. Memory 2009; 17: 26-38.

8 Raffard S, D'Argembeau A, Lardi C, Bayard S, Boulenger J, Van der Linden M. Narrative identity in schizophrenia. Conscious Cogn 2010; 19: 328-40.

9 Berna F, Bennouna-Greene M, Potheegadoo J, Verry P, Conway MA, Danion JM. Impaired ability to give a meaning to personally significant events in patients with schizophrenia. Conscious Cogn 2011; 20: 703-11

10 Cuervo-Lombard C, Jovenin N, Hedelin G, Rizzo-Peter L, Conway MA, Danion JM. Autobiographical memory of adolescence and early adulthood events: an investigation in schizophrenia. J Int Neuropsychol Soc 2007; 13: 335-43.

11 Schacter DL, Addis DR, Buckner RL. Remembering the past to imagine the future: the prospective brain. Nat Rev Neurosci 2007; 8: 657-61.

12 Van Os J, Kapur S. Schizophrenia. Lancet 2009; 374: 635-45.

13 Svoboda E, McKinnon MC, Levine B. The functional neuroanatomy of autobiographical memory: a meta-analysis. Neuropsychologia 2006; 44 2189-208

14 Cabeza R, St Jacques P. Functional neuroimaging of autobiographical memory. Trends Cogn Sci 2007; 11: 219-27.

15 Ragland JD, Laird AR, Ranganath C, Blumenfeld RS, Gonzales SM, Glahn DC. Prefrontal activation deficits during episodic memory in schizophrenia. Am J Psychiatry 2009; 166: 863-74.

16 Achim AM, Lepage M. Episodic memory-related activation in schizophrenia: meta-analysis. Br J Psychiatry 2005; 187: 500-9.

17 American Psychiatric Association. Diagnostic and Statistical Manual of Mental Disorders (4th edn, revised) (DSM-IV-TR). APA, 2000.

18 Wechsler D. The Wechsler Adult Intelligence Scale Manual (3rd edn). The Psychological Corporation, 1997.

19 Kay SR, Fiszbein A, Opler LA. The positive and negative syndrome scale (PANSS) for schizophrenia. Schizophr Bull 1987; 13: 261-76.
20 Woods Sw. Chlorpromazine equivalent doses for the newer atypical antipsychotics. J Clin Psychiatry 2003; 64: 663-7.

21 Sheehan DV, Lecrubier $Y$, Sheehan $\mathrm{KH}$, Amorim $\mathrm{P}$, Janavs J, Weiller $E$, et al. The Mini-International Neuropsychiatric Interview (M.I.N.I.): the development and validation of a structured diagnostic psychiatric interview for DSM-IV and ICD-10. J Clin Psychiatry 1998; 59: 22-33.

22 Oldfield RC. The assessment and analysis of handedness: the Edinburgh inventory. Neuropsychologia 1971; 9: 97-113.

23 Content A, Mousty P, Radeau M. Brulex. A computerized lexical database for written and spoken French [in French]. Année Psychol 1990; 4: 551-66.

24 Desrochers A, Bergeron M. Norms of subjective frequency of use and imagery for a sample of 1,916 French nouns. Can J Exp Psychol 2000; 54 274-325.

25 Piolino P, Desgranges B, Belliard S, Matuszewski V, Lalevée C, De la Sayette $\mathrm{V}$, et al. Autobiographical memory and autonoetic consciousness: triple dissociation in neurodegenerative diseases. Brain 2003; 126: 2203-19.

26 Gardiner JM, Ramponi C, Richardson-Klavehn A. Experiences of remembering, knowing, and guessing. Conscious $\operatorname{cog}$ 1998; 7: 1-26.

27 Lemogne C, Piolino P, Friszer S, Claret A, Girault N, Jouvent R, et al. Episodic autobiographical memory in depression: specificity, autonoetic consciousness, and self-perspective. Conscious Cogn 2006; 15: 258-68.

28 Friston KJ, Fletcher $\mathrm{P}$, Josephs $\mathrm{O}$, Holmes $\mathrm{A}$, Rugg MD, Turner R. Event-related fMRI: characterizing differential responses. Neuroimage 1998 7: $30-40$

29 Nichols T, Brett M, Andersson J, Wager T, Poline JB. Valid conjunction inference with the minimum statistic. Neuroimage 2005; 25: 653-60.

30 spreng RN, Mar RA, Kim ASN. The common neural basis of autobiographical memory, prospection, navigation, theory of mind, and the default mode: a quantitative meta-analysis. J Cogn Neurosci 2009; 21: 489-510.

31 Fletcher PC, Frith CD. Perceiving is believing: a Bayesian approach to explaining the positive symptoms of schizophrenia. Nat Rev Neurosci 2009; 10: $48-58$.

$32 \mathrm{Kim} \mathrm{H}$. Dissociating the roles of the default-mode, dorsal, and ventral networks in episodic memory retrieval. Neuroimage 2010; 50: 1648-57.

33 Middleton FA, Strick PL. Anatomical evidence for cerebellar and basal ganglia involvement in higher cognitive function. Science 1994; 266: 458-61.

34 Middleton FA, Strick PL. Cerebellar projections to the prefrontal cortex of the primate. J Neurosci 2001; 21: 700-12.

35 Becker S, Lim J. A computational model of prefrontal control in free recall: strategic memory use in the California verbal learning task. J Cogn Neurosci 2003; 15: 821-32.

36 Rekkas PV, Constable RT. Evidence that autobiographic memory retrieval does not become independent of the hippocampus: an fMRI study contrasting very recent with remote events. J Cogn Neurosci 2005; 17 1950-61.

37 Söderlund H, Moscovitch M, Kumar N, Mandic M, Levine B. As time goes by: hippocampal connectivity changes with remoteness of autobiographical memory retrieval. Hippocampus 2012; 22: 670-9.

38 Lodge DJ, Grace AA. Divergent activation of ventromedial and ventrolateral dopamine systems in animal models of amphetamine sensitization and schizophrenia. Int J Neuropsychopharmacol 2011; 18: 1-8.

39 Minzenberg MJ, Laird AR, Thelen S, Carter CS, Glahn DC. Meta-analysis of 41 functional neuroimaging studies of executive function in schizophrenia. Arch Gen Psychiatry 2009; 66: 811-22. 